\title{
Increased Expression of Mitochondrial-encoded Genes in Skeletal Muscle of Humans with Diabetes Mellitus
}

\author{
David A. Antonetti, Christine Reynet, and C. Ronald Kahn \\ Research Division, Joslin Diabetes Center and Department of Medicine, Harvard Medical School, Boston, Massachusetts 02215
}

\begin{abstract}
Screening subtraction libraries from normal and type II diabetic human skeletal muscle, we identified four different mitochondrially encoded genes which were increased in expression in diabetes. The genes were cytochrome oxidase I, cytochrome oxidase III, NADH dehydrogenase IV, and 12s rRNA, all of which are located on the heavy strand of the mitochondrial genome. There was a 1.5- to 2.2-fold increase in the expression of these mRNA molecules relative to total RNA in both type I and type II diabetes as assessed by Northern blot analyses. Since there was $\sim 50 \%$ decrease in mitochondrial DNA copy number as estimated by Southern blot analyses, mitochondrial gene expression increased $\sim$ 2.5-fold when expressed relative to mitochondrial DNA copy number. For cytochrome oxidase I similar changes in mitochondrial gene expression were observed in muscle of nonobese diabetic and ob/ob mice, models of type $I$ and type II diabetes, respectively. By contrast there was no change or a slight decrease in expression of cytochrome oxidase 7a, a nuclear-encoded subunit of cytochrome oxidase, and the expression of mitochondrial transcription factor 1 in human skeletal muscle did not change with type I or type II diabetes. The increased mitochondrial gene expression may contribute to the increase in mitochondrial respiration observed in uncontrolled diabetes. (J. Clin. Invest. 1995. 95:13831388.) Key words: mitochondria • insulin • electron transport $\bullet$ oxidative phosphorylation $\cdot$ mice
\end{abstract}

\section{Introduction}

Mitochondria contain closed circular, double-stranded DNA of $\sim 16.5 \mathrm{~kb}$. Both strands of the mitochondrial DNA are tran-

Address correspondence to C. Ronald Kahn, Joslin Diabetes Center, Research Division, One Joslin Place, Boston, MA 02215. Phone: 617732-2642; FAX: 617-732-2593.

Received for publication 8 March 1994 and in revised form 7 October 1994.

1. Abbreviations used in this paper: Cox $7 \mathrm{aM}$, cytochrome oxidase $7 \mathrm{a}$ muscle isoform; Cox I, cytochrome oxidase I; Cox III, cytochrome oxidase III; H-strand, heavy strand of mitochondrial DNA; mtTF1, mitochondrial transcription factor 1; NOD, nonobese diabetic; STZ, streptozotocin diabetic animal model.

J. Clin. Invest.

(C) The American Society for Clinical Investigation, Inc.

0021-9738/95/03/1383/06 \$2.00

Volume 95, March 1995, 1383-1388 scribed. The heavy $(\mathrm{H})^{1}$ strand encodes genes for $12 \mathrm{~s}$ and $16 \mathrm{~s}$ mitochondrial ribosomal RNA, 12 polypeptides, and 14 transfer RNAs, while the light strand encodes one polypeptide and 8 transfer RNAs. The polypeptides are translated by mitochondrial ribosomes and are components of the electron transport chain (for review see references 1-4). It is believed that as with nuclear-encoded genes, mitochondrial gene transcription is regulated through trans-acting factors binding cis elements, although only a limited number of the trans elements which control these processes have been identified. The only transcription factor which has been cloned is the nuclear-encoded mitochondrial transcription factor 1 (mtTF1) (5). In addition, and as opposed to nuclear-encoded genes, mitochondrial-encoded gene expression may also be regulated by changes in mitochondrial DNA copy number.

Both type I (insulin-dependent) and type II (non-insulindependent ) diabetes mellitus are characterized by alterations in glucose, protein, and lipid metabolism in skeletal muscle $(6,7)$. These alterations are the result of changes in gene expression, as well as posttranslational modifications of proteins. Insulin itself has been shown to regulate expression of a large number of genes (for reviews see Meisler and Howard [8] and O'Brien and Granner [9]); however, relatively little is known about insulin or diabetes-regulated gene expression in skeletal muscle. In an effort to identify changes in gene expression which might contribute to the pathogenesis of this disease, we have screened subtraction libraries made from normal and type II diabetic muscle tissue (10). This report details our identification of four mitochondrial-encoded genes whose expression was altered in diabetes, and our efforts to characterize the mechanisms responsible for the observed changes.

\section{Methods}

Screening normal-enriched and diabetes-enriched libraries. Subtraction libraries were made using cDNA generated from human leg skeletal muscle RNA isolated from normal and type II diabetic patients undergoing amputation (10). Individual clones in these subtraction libraries were grown in 96-well dishes and screened for differential regulation in diabetes as described by Kahn and Reynet (11). Briefly, clones from the normal-enriched and diabetic-enriched libraries were transformed into XL-1 blue supercompetent cells (Stratagene Inc., La Jolla, CA), and the cells were grown on Luria-Bertani plates containing ampicillin. Colonies were picked using a sterile toothpick and used to inoculate separate wells of 96-well plates (Falcon Labware, Becton Dickinson, Lincoln Park, NJ) containing Luria-Bertani ampicillin media. The plates were grown overnight at $37^{\circ} \mathrm{C}$ in a shaking incubator. Using beaded lids (Falcon Labware), replica plates were made of the 96-well plates. Glycerol was added to each well of the archive plates to give a final concentration of $20 \%$, and these archive plates were stored at $-85^{\circ} \mathrm{C}$. The replica plates were used to make duplicate dot blots on 
Biotrans nylon membranes (ICN Biomedicals, Inc., Costa Mesa, CA) using $20 \mu \mathrm{l}$ of cell culture per well. The membranes were denatured with $0.5 \mathrm{~N} \mathrm{NaOH}$ for $7 \mathrm{~min}$, neutralized with $1 \mathrm{M}$ Tris $\mathrm{pH} 7.4$, for 4 min, and rinsed with $10 \times$ SSC for $4 \mathrm{~min}$. The DNA was cross-linked to the membranes with ultraviolet light. The blots were rinsed in $2 \times$ SSC for $2 \mathrm{~min}$, wrapped in Saran wrap, and stored at $4^{\circ} \mathrm{C}$.

Subtracted probes were prepared from the normal-enriched and diabetic-enriched libraries using PCR followed by EcoRI digestion and a multiprime labeling reaction (11). Free nucleotides were separated from the probes using Elutips (Schleicher and Schuell, Inc., Keene, NH). After a prehybridization of at least $3 \mathrm{~h}$ to reduce nonspecific binding, the dot blots were hybridized for at least $24 \mathrm{~h}$ with the labeled probes in $50 \%$ formamide, $5 \times \mathrm{SSC}, 0.1 \%$ SDS, $5 \times$ Denhardt's plus $100 \mu \mathrm{g} /$ $\mathrm{ml}$ salmon sperm DNA, and $1 \mu \mathrm{g} / \mathrm{ml} \mathrm{KpnI} \mathrm{cut} \mathrm{pBluescript} \mathrm{(Stratagene,}$ Inc.). One of the duplicate blots was hybridized with the normal-enriched library probe and the other duplicate blot was hybridized with the diabetic-enriched library probe. Equal counts of the normal-enriched and diabetic-enriched library probes were added $\left(\sim 1 \times 10^{6} \mathrm{cpm} / \mathrm{ml}\right)$. Prehybridization and hybridization were at $42^{\circ} \mathrm{C}$. The blots were washed two times for 20 min each at room temperature with $2 \times$ SSC, $1 \%$ SDS, and one time for $20 \mathrm{~min}$ at $60^{\circ} \mathrm{C}$ in $0.2 \times \mathrm{SSC}, 1 \% \mathrm{SDS}$, and analyzed by autoradiography.

Characterization of clones. Clones of genes which were shown to be differentially regulated by the above screening method were grown in LB ampicillin media and purified using a plasmid purification system (QIAGEN, Chatsworth, CA). Sequencing was done using Sequenase Version 2.0 sequencing kit (United States Biochemical Corp., Cleveland, $\mathrm{OH}$ ). The sequencing reaction products were separated on a $6 \%$ polyacrylamide gel (Sequagel; National Diagnostics, Inc., Atlanta, GA).

Patients. Mixed fiber type human muscle samples were obtained from patients undergoing amputation. The patients in the control, type I, and type II diabetic groups were both male and female of various ages. Samples were taken from either gastrocnemius or quadracep muscle. No systematic change in GLUT4 mRNA levels were observed (Antonetti, D. A., C. Reynet, and C. R. Kahn, unpublished observations).

Northern blot analysis. Human leg, skeletal muscle RNA was isolated using RNAzol B (Biotecx Laboratories, Inc., Houston, TX). RNA was also isolated from nonobese diabetic (NOD) mice which were diabetic for $\sim 1$ mo and from age-matched controls using Snap-o-Sol (Biotecx) and from experimentally induced diabetic rats using RNAzol B (Biotecx). Experimentally induced diabetic rats were obtained by one intraperitoneal streptozotocin (Sigma Chemical Co., St. Louis, MO) injection $(100 \mathrm{mg} / \mathrm{kg}$ body wt in citrate buffer $\mathrm{pH} 4.5)$ and were studied 1 wk after injection. The RNA was separated by electrophoresis on formaldehyde-agarose gels and transferred to nylon membranes by blotting (12). Blots were hybridized in $50 \mathrm{mM}$ Pipes ( $\mathrm{pH} 6.5$ ), $100 \mathrm{mM}$ $\mathrm{NaCl}, 50 \mathrm{mM}$ sodium phosphate, $1 \mathrm{mM}$ EDTA, 5\% SDS, and $100 \mu \mathrm{g} /$ $\mathrm{ml}$ salmon sperm DNA at $42^{\circ} \mathrm{C}$ after a prehybridization of at least $3 \mathrm{~h}$. The blots were washed three times for 15 min each at $65^{\circ} \mathrm{C}$ in $5 \% \mathrm{SDS}$, $0.5 \times \mathrm{SSC}$ and one time for $15 \mathrm{~min}$ at $65^{\circ} \mathrm{C}$ in $0.1 \% \mathrm{SDS}, 0.2 \times \mathrm{SSC}$. The blots were analyzed either by autoradiography and densitometric scanning or by using a Phosphorimager (Molecular Dynamics, Inc., Sunnyvale, CA) and quantitated using Imagequant Software (Molecular Dynamics, Inc.). For all Northern blots using human RNA, a single sample from one normal individual was used as an internal control for normalization of the data. Nearly all blots used were stripped and reprobed with clones whose expression were unchanged in type $I$ and type II diabetes to ensure equal loading of RNA samples.

Quantitation of mitochondrial DNA copy number. Total genomic DNA was isolated from leg skeletal muscle of normal, type I, and type II diabetic human patients undergoing amputation. Frozen tissue samples were crushed using a mortar and pestle cooled with liquid $\mathrm{N}_{2}$. For every $100 \mathrm{mg}$ tissue, $1.2 \mathrm{ml}$ of digestion buffer $(100 \mathrm{mM} \mathrm{NaCl}, 10 \mathrm{mM}$ Tris pH 7.5, $25 \mathrm{mM}$ EDTA, $0.5 \%$ SDS, and freshly added $0.1 \mathrm{mg} / \mathrm{ml}$ proteinase $\mathrm{K}$ ) was added. The samples were incubated with shaking at $50^{\circ} \mathrm{C}$ for $18 \mathrm{~h}$. The samples were extracted twice with an equal volume of phenol/chloroform/isoamylalcohol $(25 / 24 / 1)$ then once with an equal volume of ether. The DNA was precipitated from solution at room

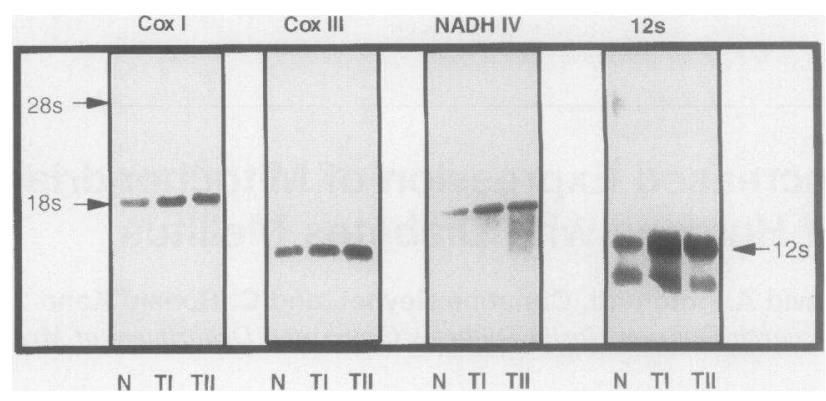

Figure 1. Northern blot analyses using clones of mitochondrial-encoded genes isolated from subtracted libraries. A composite figure was made depicting typical Northern analyses of mitochondrial-encoded genes and the effect of diabetes. RNA was isolated from human skeletal muscle, separated by gel electrophoresis, and blotted onto nylon membranes. Each set of three lanes represents a normal $(N)$, type $I(T I)$, and type II diabetic (TII) patient, respectively. From left to right the blots were probed with radiolabeled fragments of Cox I, Cox III, NADH dehydrogenase IV, and 12s rRNA.

temperature by adding $0.5 \mathrm{vol}$ of $7.5 \mathrm{M}$ ammonium acetate and 2 vol of ethanol. The strands of DNA were fished from solution using a glass pipette hook, rinsed in $70 \%$ ethanol, allowed to dry, and resuspended in Tris-EDTA. The concentration of DNA was determined spectrophotometrically. The DNA was digested with one of four enzymes: EcoRI, PstI, BamHI, or KpnI. Southern blot analyses were then performed essentially as described in Sambrook et al. (13), and the results were quantitated using a Phosphoimager and ImageQuant software. The value for each sample was normalized to one control patient as described above.

Plasmids. Plasmid mtTF1 was obtained from Dr. D. A. Clayton, Stanford University School of Medicine, Stanford, CA and contained nucleotides $259-873$ of human mitochondrial transcription Factor 1 plus an additional 5' ATG in pBluescript II KS (+) (5). Plasmid pCox7aM.541 containing a 341-bp insert coding for the full-length human cytochrome oxidase VIIa-muscle isoform cDNA in vector $\mathrm{pBSt}$ was kindly provided by Dr. E. A. Schon, Columbia University College of Physicians and Surgeons, New York (14).

Statistical analyses. For statistical analyses, a Student's $t$ test was used with a two-tailed $P$ value.

\section{Results}

Identifying clones from the subtraction libraries which are differentially expressed in diabetes. Subtraction libraries made from human skeletal muscle RNA were screened with subtracted probes to identify clones which encode genes differentially expressed in diabetes as described in Methods $(10,11)$. The presumptive differentially expressed clones were then used as probes in Northern blot analyses of human skeletal muscle to determine more precisely the effect of type I and type II diabetes on expression of the genes encoded by the clones. Of over 5,000 clones screened, 19 were found to be differentially expressed in type I and/or type II diabetes. These clones were partially sequenced and compared to the data banks of known genes. Using these techniques, four mitochondrial-encoded genes were identified which were increased in expression in type I and type II diabetes. They are cytochrome oxidase I (Cox I), cytochrome oxidase III (Cox III), NADH dehydrogenase IV, and 12s rRNA. As compared to the nondiabetic, the expression of these clones was increased 1.55- to 1.94-fold at the RNA level (Fig. 1 and Table I). More extensive Northern blot 


\begin{tabular}{|c|c|c|c|c|}
\hline \multirow[b]{2}{*}{ Gene } & \multirow[b]{2}{*}{ Insert size } & \multirow[b]{2}{*}{ RNA size } & \multicolumn{2}{|c|}{ Expression in type II diabetes } \\
\hline & & & per total RNA & per mtDNA \\
\hline Cytochrome oxidase I* & $801 ; 646$ bp & $1.6 \mathrm{~kb}$ & $1.55 \mathrm{X}$ & $2.49 \mathrm{X}$ \\
\hline Cytochrome oxidase III & $273 \mathrm{bp}$ & $1.0 \mathrm{~kb}$ & $1.69 \mathrm{X}$ & $3.01 \mathrm{X}$ \\
\hline NADH dehydrogenase IV* & $423 ; 1240 \mathrm{bp}$ & $1.4 \mathrm{~kb}$ & $1.94 \mathrm{X}$ & $2.13 \mathrm{X}$ \\
\hline $12 \mathrm{~s}$ rRNA & $400 \mathrm{bp}$ & $1.0 \mathrm{~kb}$ & $1.78 \mathrm{X}$ & $2.54 \mathrm{X}$ \\
\hline
\end{tabular}

Subtraction cDNA libraries made from normal and type II diabetic skeletal muscle RNA were screened for clones which encoded genes differentially expressed in diabetes vs normal conditions. Clones of genes which were differentially regulated were sequenced and compared to data banks of known genes. Four mitochondrial-encoded genes were identified; all were increased in expression in both type I and type II diabetes. * NADH dehydrogenase IV and Cox I were identified in two independent clones.

analyses were done for the clones encoding Cox I, Cox III, and 12s rRNA (Fig. 2 and Table I). The expression of RNA for all of these genes was increased to a similar extent in both type I and type II diabetes as compared to normal. This increase was

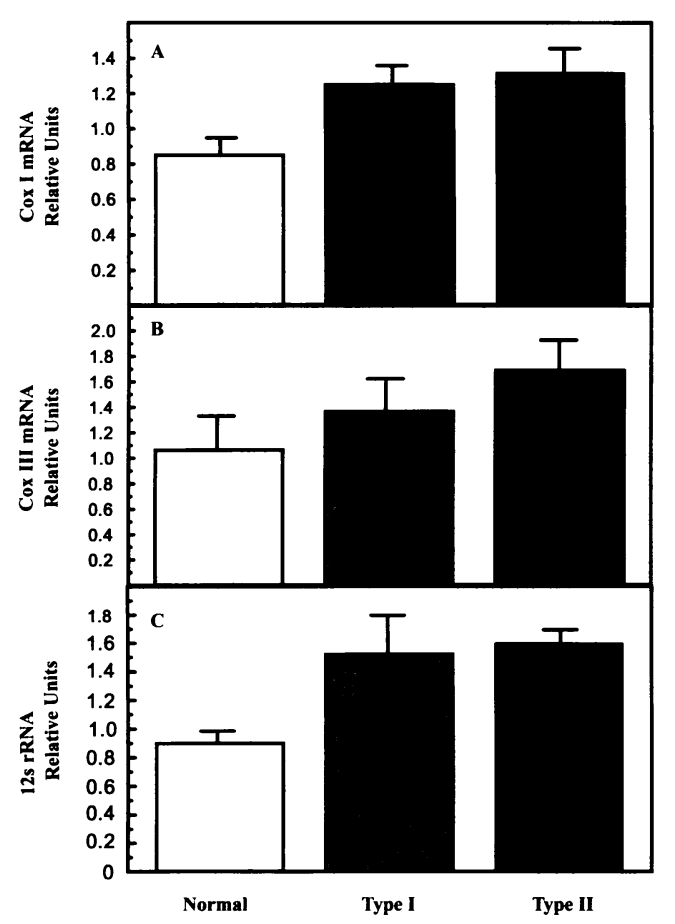

Figure 2. Effect of diabetes on cytochrome oxidase I mRNA, cytochrome oxidase III mRNA, and 12s rRNA expression. The effect of diabetes on mitochondrial-encoded RNA expression in human skeletal muscle was measured by Northern blot analysis. The level of expression was quantitated using a Phosphoimager or autoradiography and scanning densitometry. All results were normalized to the same nondiabetic patient used on all blots. Results are presented as the mean \pm SEM. $(A)$ Expression of cytochrome oxidase I mRNA. The $P$ value for type I (four determinations in three patients) vs normal (five determinations in three patients) is 0.03 . The $P$ value for type II (five determinations in three patients) vs normal is 0.02. ( $B$ ) Expression of cytochrome oxidase III mRNA. For control and type I patients, four determinations from three patients were made; for type II patients five determinations from four patients were made. These values were not significantly different. $(C$ ) Expression of $12 \mathrm{~s}$ rRNA. The $P$ value for type I (four determinations in two patients) vs normal (four determinations in three patients) is 0.06 . The $P$ value for type II $(n=4)$ vs normal is 0.0007 . 1.55-fold for Cox I, 1.69-fold for Cox III, and 1.79-fold for 12s rRNA and was statistically significant $(P \leq 0.02)$ for Cox I and $12 \mathrm{~s}$ rRNA.

Effect of diabetes on mitochondrial DNA copy number. To determine the mitochondrial DNA copy number, Southern blot analyses were performed on DNA from human skeletal muscle. Since mitochondrial DNA is only $1 \%$ of total DNA (15), total genomic DNA was isolated from human skeletal muscle and digested by four different enzymes, separated by gel electrophoresis, blotted onto nylon membranes, and probed with a radiolabeled Cox I probe to estimate mitochondrial DNA copy number. As expected PstI digestion yielded two fragments while EcoRI, BamHI, and KpnI yielded one fragment each. Surprisingly, as shown by the intensity of hybridization for all five fragments,

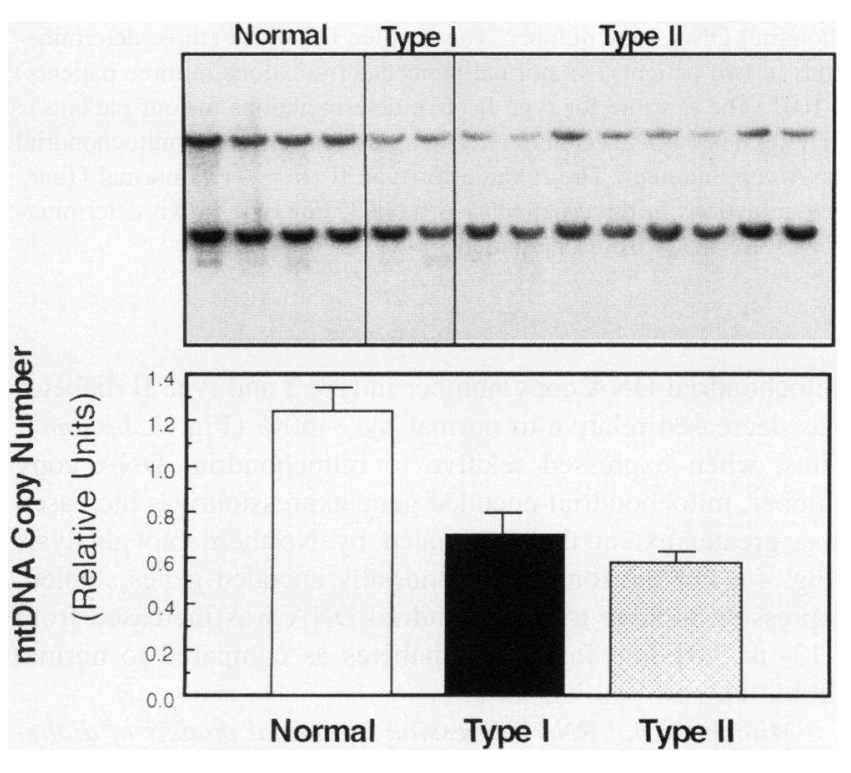

Figure 3. Effect of diabetes on mitochondrial DNA copy number. Total cellular DNA was digested with restriction enzymes EcoRI, PstI,

BamHI, and KpnI. The DNA was separated by gel electrophoresis and blotted onto nitrocellulose. The blots were probed with radiolabeled Cox I fragment. (Top) Southern blot. Phosphorimage of the resultant Southern blot after Pstl digestion. (Bottom) Quantitation of Southern blots. The bands were quantitated using a Phosphorimager and normalized to one normal patient. Shown are the means \pm SEM. The $P$ value for type I $(n=10)$ vs normal $(n=20)$ is 0.006 . The $P$ value for type II $(n=40)$ vs normal is $<0.0001$. 


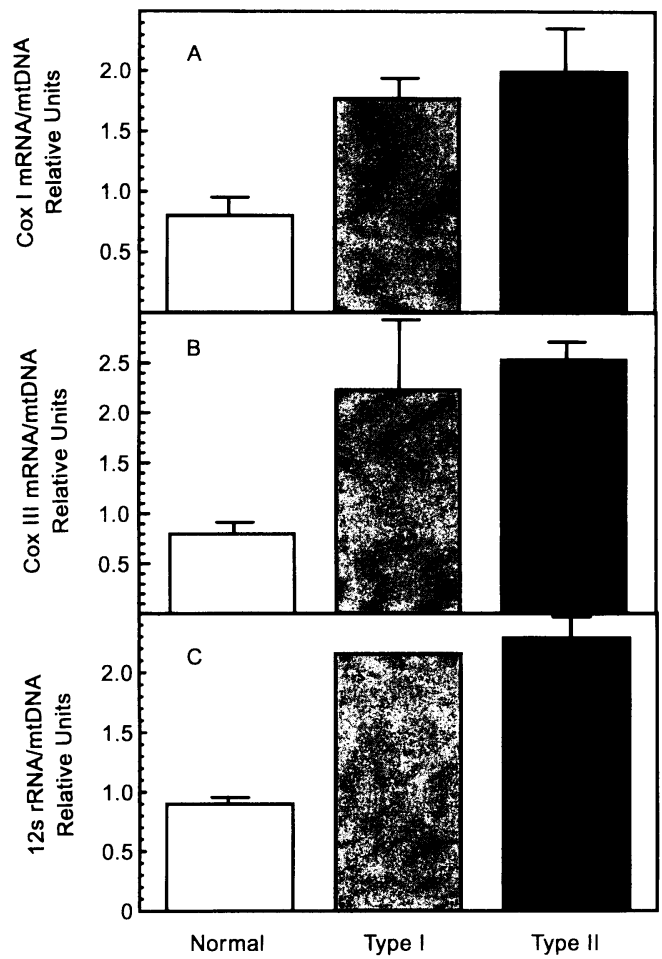

Figure 4. Expression of RNA relative to mtDNA copy number. The expression of RNA was normalized to the mitochondrial DNA copy number for that patient. Data are presented as the mean \pm SEM. $(A)$ Cytochrome oxidase I mRNA expression relative to mitochondrial DNA copy number. The $P$ value for type I ( three determinations in two patients) vs normal (five determinations in three patients) is 0.005 . The $P$ value for type II ( five determinations in three patients) vs normal is 0.02. (B) Cytochrome oxidase III mRNA expression relative to mitochondrial DNA copy number. The $P$ value for type I (three determinations in two patients) vs normal (four determinations in three patients) is 0.07 . The $P$ value for type II (five determinations in four patients) vs normal is 0.0002 . ( $C$ ) 12s rRNA expression relative to mitochondrial DNA copy number. The $P$ value for type II $(n=4)$ vs normal (four determinations in three patients) is 0.0003 . For type I, two determinations were made from one patient.

mitochondrial DNA copy number in type I and type II diabetes was decreased relative to normal by $\sim 50 \%$ (Fig. 3, bottom). Thus, when expressed relative to mitochondrial DNA copy number, mitochondrial-encoded gene expression was increased to a greater extent than estimated by Northern blot analysis (Fig. 4). For the four mitochondrially encoded genes studied, expression relative to mitochondrial DNA was increased from 2.13- to 3.01-fold in type II diabetes as compared to normal ( Table I).

Mitochondrial RNA expression in animal models of diabetes. The effect of diabetes on Cox I and Cox III expression in skeletal muscle of NOD mice, a type I diabetic animal model, was determined by Northern blot analysis. As in humans with type I diabetes, the expression of Cox I mRNA increased 1.67fold in the diabetic animals as compared to controls while Cox III expression increased 1.35-fold (Fig. 5). An increase of 1.4fold in the expression of Cox I mRNA was also observed in the skeletal muscle of ob/ob mice, a model of type II diabetes, relative to the control ob/ + mice (Fig. 6). Interestingly, skeletal muscle of rats with 1 wk of streptozotocin-induced type I diabe-

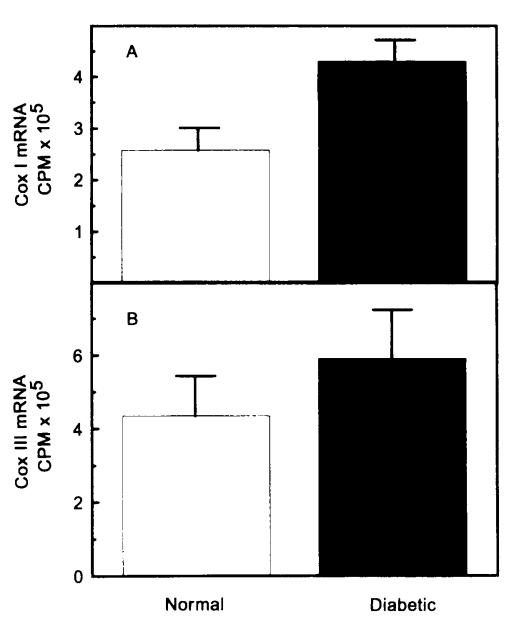

Figure 5. Cytochrome oxidase I and cytochrome oxidase III mRNA expression in NOD mice. The level of cytochrome oxidase I and cytochrome oxidase III mRNA in skeletal muscle of NOD mice was measured by Northern blot analysis. RNA was isolated from quadriceps of normal and agematched controls, separated by gel electrophoresis, blotted onto nylon membranes, and probed with either a radiolabeled Cox 1 fragment $(A)$ or a radiolabeled Cox III fragment $(B)$. Phosphoimaging was used to quantitate the blots. The results are presented as the mean \pm SEM. The $P$ value for the diabetic $(n=3)$ vs the normal $(n=3)$ mice is 0.04 for Cox I, but was not significantly different for Cox III.

tes did not show a change in Cox I mRNA expression in repeated experiments, suggesting that $1 \mathrm{wk}$ of diabetes may not be long enough to observe changes in Cox I gene expression in skeletal muscle. However, an increase in expression of Cox I mRNA could be observed in liver and cardiac muscle of streptozotocin diabetic rats as compared to controls (Fig. 6).

Diabetes and cytochrome oxidase 7 a muscle isoform and mitochondrial transcription Factor I mRNA expression. To elucidate the molecular mechanism by which diabetes might regulate mitochondrial gene expression and mitochondrial DNA copy number, we examined the effect of diabetes on the mRNA level of the nuclear-encoded cytochrome oxidase subunit, cytochrome oxidase 7 a muscle isoform $(\mathrm{Cox} 7 \mathrm{aM})$ and the nuclearencoded mitochondrial transcription factor $\mathrm{mtTF} 1$. In human skeletal muscle the mRNA of Cox $7 \mathrm{aM}$ was not increased in diabetes. Fig. 7 is a representative result of four Northern or slot blot hybridization experiments in which no consistent change in
A STZ Diabetic Rat Skeletal Muscle

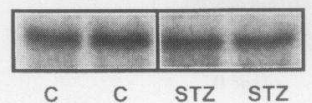

B

STZ Diabetic Rat Heart and Rat Liver
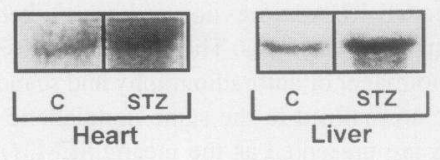

C Obese Mouse

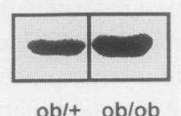

Figure 6. Cytochrome oxidase I expression in streptozotocin diabetic rats and genetically obese mice. Northern blot analyses were used to determine the effect of diabetes on Cox I expression in streptozotocin diabetic rats and obese mice. (A) Effect of diabetes on Cox 1 expression in streptozotocin rat skeletal muscle. No change in expression was observed as compared to normal rats. $(B)$ Diabetes caused an increase of 2.51-fold in cardiac muscle and 2.05-fold in liver of streptozotocin diabetic rats as compared to normal rats. (C) Cox I mRNA expression was increased 1.40-fold in a genetically obese mouse as compared to control. STZ, streptozotocin diabetic rat. 


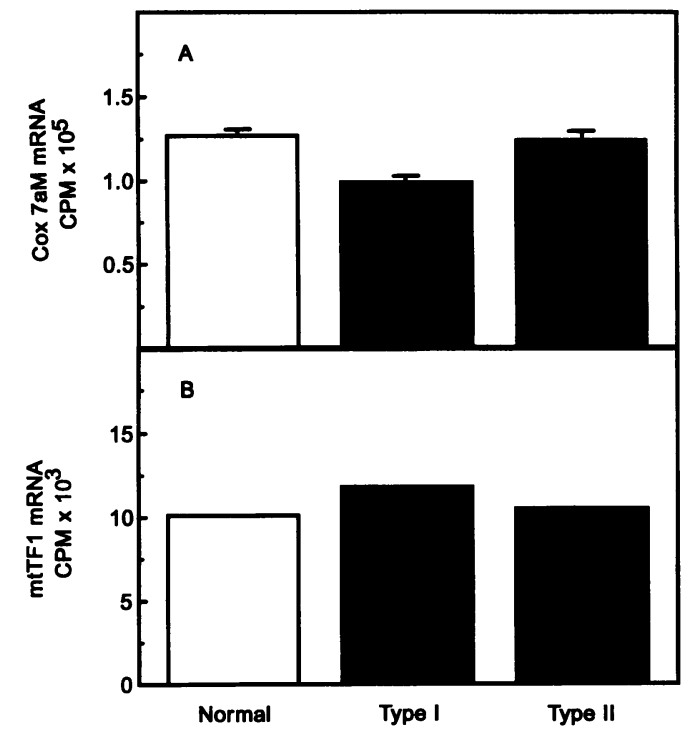

Figure 7. Effect of diabetes on expression of nuclear-encoded cytochrome oxidase 7a muscle isoform and mitochondrial transcription factor 1. (A) Northern and slot blot analysis was used to determine mRNA expression of $\operatorname{Cox} 7 \mathrm{aM}$ in human skeletal muscle of diabetic and normal patients. Presented is a representative slot blot analysis in which the average cpm of quadruplicate slots from one normal, one type I, and one type II diabetic patient is revealed. No consistent change in Cox $7 \mathrm{aM}$ expression was observed in this experiment nor in three other hybridization analyses. $(B)$ Slot blot analysis using total RNA from human skeletal muscle was used to determine the effect of diabetes on mitochondrial transcription factor 1 expression. Duplicate slots of one normal, one type I, and one type II patient were made. The blot was probed with a radiolabeled mtTF1 fragment and the data are presented as the average cpm for each condition.

Cox 7aM expression was observed. Using slot blot analysis of human skeletal muscle RNA, we found no difference in the level of mtTF1 mRNA between normal, type I, and type II diabetic patients (Fig. 7).

\section{Discussion}

To identify changes in gene expression which might contribute to the pathogenesis of human diabetes mellitus, subtraction libraries made from normal and type II diabetic human skeletal muscle were screened using subtractive probes. Using this approach we identified four mitochondrial encoded genes: cytochrome oxidase I, cytochrome oxidase III, NADH dehydrogenase IV, and 12s rRNA, whose expression is increased in both type I and type II diabetes. This occurs despite a decrease in mitochondrial DNA copy number and with no change in the nuclear-encoded cytochrome oxidase VIIaM subunit or transacting factor mitochondrial transcription factor 1 . All of the mitochondrial-encoded genes studied are located on the mitochondrial $\mathrm{H}$-strand. The $\mathrm{H}$-strand has two sites where transcription is initiated (16). Initiation from one site yields a transcript of the entire heavy strand and is processed to the individual mRNAs, tRNAs, and rRNAs; initiation from the other site transcribes the rRNAs and a limited number of tRNAs. The fact that the three mRNAs and one rRNA increase in expression to a similar extent, suggests that the effect of diabetes on mitochondrial gene expression is mediated through complete heavy chain transcription. The effect of diabetes on light strand transcription was not studied.

Previously, Williams et al. $(17,18)$ proposed that the major mechanism by which mitochondrial gene expression is regulated is through changes in mitochondrial DNA copy number. Changes in oxidative capacity of different skeletal muscle fiber types correlates with differences in mitochondrial DNA copy number (17), and exercise-induced changes in oxidative capacity also correlates with changes in mitochondrial DNA copy number $(17,18)$. In the present study, increased mitochondrial gene expression occurs under circumstances in which the mitochondrial DNA copy number is decreased. A similar discordance between mitochondrial gene regulation and DNA copy number has been reported by Van Itallie who found increased oxidative capacity in a hepatoma cell line after thyroid hormone addition and mitochondrial gene expression, but no change in mitochondrial DNA copy number (19).

All of the known trans elements controlling mitochondrial transcription and replication in humans are nuclear encoded. The transcription factor mtTFl binds both $\mathrm{H}$-strand and light strand promoters, and its DNA binding has been correlated with the ability of in vitro extracts to initiate transcription (3). mtTF1 may have multiple roles in initiation of DNA replication, both as a factor initiating primer transcription and as a factor involved in processing the primer (3). In diabetes, the change in mitochondrial gene expression is not regulated by a change in DNA copy number or by a change in expression of mtTF1. However, it is possible that a posttranslational modification of mtTF1 or the expression or modification of another trans-acting factor is involved in the regulation of mitochondrial gene expression in diabetes.

In this study we also measured the mRNA of a nuclearencoded mitochondrial enzyme Cox $7 \mathrm{aM}$ to determine if changes in nuclear-encoded mitochondrial genes are coordinated with changes in mitochondrial encoded genes. A number of the nuclear-encoded mitochondrial enzymes contain a binding site for nuclear regulatory factor 1 which may be involved in gene regulation. A binding site for nuclear regulatory factor 1 has also been identified in mitochondrial RNA processing RNA, a factor involved in mitochondrial DNA replication (20, 21 ). Other cis elements common to mitochondrial genes have been identified (22). We found that in diabetes, the level of Cox 7aM mRNA was unchanged or possibly slightly decreased. Again, this is similar to the effect of thyroid hormone in cell culture in that changes in mitochondrial DNA gene expression are not necessarily coordinated with changes in nuclear-encoded mitochondrial enzymes $(19,23,24)$. Increases in electron transport chain complexes may be achieved under such circumstances by a pool of unutilized nuclear-encoded subunits. For example, it has been proposed that mitochondrial-encoded Cox I regulates cytochrome $c$ oxidase assembly and the nuclearencoded subunits are not limiting $(19,23,25)$.

There have been a number of case studies defining a link between point mutations $(26,27)$, duplication $(28)$, and deletion (29) of mitochondrial genes with diabetes; (for review see Gerbitz [30]). In all these cases, the patients have multisystem genetic disorder of which diabetes is only one component. To our knowledge, the only studies on mitochondrial gene expression in diabetes are those of Minchenko who found a decrease in mitochondrial gene expression in liver and heart of rats with experimentally induced diabetes which could be reversed by insulin treatment $(31,32) .3-4$ wk of streptozotocin induced 
diabetes also results in a decrease in mitochondrial oxidative phosphorylation in heart by $\sim 50 \%$ (33) and a $\sim 50 \%$ decrease in oxidative phosphorylation in skeletal muscle (34). By contrast, human skeletal muscle mitochondria from well controlled diabetics show no change in oxidative phosphorylation, and in uncontrolled diabetes there is an increase in mitochondrial respiration by $\sim 1.50$-fold (35). This is in agreement with our finding of an increase in expression of mitochondrially encoded genes of the oxidative phosphorylation pathway. Furthermore, in rats with long-term alloxan or streptozotocin-induced diabetes, mitochondrial oxidative phosphorylation is increased $\sim$ 1.40-fold in liver (36).

In summary, diabetes mellitus increases skeletal muscle mitochondrial gene expression despite decreased mitochondrial DNA copy number. The effect of diabetes on mitochondrial gene expression is mediated by an as yet undefined mechanism, but does not appear to involve mtTF1 expression. The change in mitochondrial gene expression could contribute to changes in oxidative phosphorylation observed in uncontrolled diabetes in humans and some diabetic animal models.

\section{Acknowledgments}

We thank Dr. Allison Goldfine for providing genomic DNA samples for Southern blot analyses.

This research was funded by an endocrinology training grant awarded to Brigham and Women's Hospital, the Joslin Diabetes Center (2 T32 DK 07337), National Institutes of Health grant DK 33201 (C. R. Kahn), and a grant from The Juvenile Diabetes Foundation to C. Reynet.

\section{References}

1. Wallace, D. C. 1992. Diseases of the mitochondrial DNA. Annu. Rev Biochem. 61:1175-1212.

2. Clayton, D. A. 1991. Replication and transcription of vertebrate mitochondrial DNA. Annu. Rev. Cell Biol. 7:453-478.

3. Clayton, D. A. 1991. Nuclear gadgets in mitochondrial DNA replication and transcription. TIBS (Trends Biochem. Sci.). 16:107-111.

4. Attardi, G. 1988. Biogenesis of mitochondria. Annu. Rev. Cell Biol. 4:289333.

5. Parisi, M. A., and D. A. Clayton. 1991. Similarity of human mitochondrial transcription factor 1 to high mobility group proteins. Science (Wash. DC). 252:965-969.

6. Beck-Nielsen, H., A. Vaag, and P. Damsbo. 1992. Insulin resistance in skeletal muscles in patients with NIDDM. Diabetes Care. 15:418-429.

7. Jefferson, L. S. 1979. Role of insulin in the regulation of protein synthesis Diabetes. 29:487-496.

8. Meisler, M. H., and G. Howard. 1989. Effects of insulin on gene transcription. Annu. Rev. Physiol. 51:701-714.

9. O'Brien, R. M., and D. K. Granner. 1991. Review article, regulation of gene expression by insulin. Biochem. J. 278:609-619.

10. Reynet, C., and C. R. Kahn. 1993. Rad: a member of the ras family overexpressed in muscle of type II diabetic humans. Science (Wash. DC). 262:1441-1444.

11. Kahn, C. R., and C. Reynet. 1994. Identification of diabetes-related genes by subtraction cloning. In Molecular Biology of Diabetes, Part 2. B. Draznin and D. LeRoith, editors. The Human Press Inc., Totowa, NJ. In press.

12. Lehrach, H. 1977. RNA molecular weight determinations by gel electro- phoresis under denaturing conditions, a critical re-examination. Biochemistry. 16:4743-4751.

13. Sambrook, J., E. F. Fritsch, and T. Maniatis. 1989. Molecular Cloning: A Laboratory Manual. 2nd ed. Cold Spring Harbor Laboratory Press, Cold Spring Harbor, NY.

14. Arnaudo, E., M. Hirano, R. S. Seelan, A. Milatovich, C. L. Hsieh, G. M. Fabrizi, L. I. Grossman, U. Francke, and E. A. Schon. 1992. Tissue-specific expression and chromosome assignment of genes specifying two isoforms of subunit VIIa of human cytochrome c oxidase. Gene (Amst.). 119:299-305.

15. Alberts, B., D. Vray, S. Lewis, M. Raff, K. Roberts, and J. D. Watson 1989. Molecular Biology of the Cell. Garland Publishing Inc., New York. 1146 pp.

16. Montoya, J., G. L. Gaines, and G. Attardi. 1983. The pattern of transcription of the human mitochondrial rRNA genes reveals two overlapping transcription units. Cell. 34:151-159.

17. Williams, R. S. 1986. Mitochondrial gene expression in mammalian striated muscle. J. Biol. Chem. 261:12390-12394.

18. Williams, R. S., S. Salmons, E. A. Newsholme, R. E. Kaufman, and J. Mellor. 1986. Regulation of nuclear and mitochondrial gene expression by contractile activity in skeletal muscle. J. Biol. Chem. 261:376-380.

19. Van Itallie, C. M. 1990. Thyroid hormone and dexamethasone increase the levels of a messenger ribonucleic acid for a mitochondrially-encoded subunit but not for a nuclear-encoded subunit of cytochrome c oxidase. Endocrinology. 127:55-62.

20. Evans, M. J., and R. C. Scarpulla. 1990. NRF-1: A trans-activator of nuclear-encoded respiratory genes in animal cells. Genes \& Dev. 4:1023-1034.

21. Ching-man, A. C., M. J. Evans, and R. C. Scarpulla. 1992. Nuclear respiratory factor 1 activation sites in genes encoding the gamma-subunit of ATP synthase, eukaryotic initiation factor 2 -alpha, and tyrosine aminotransferase. $J$. Biol. Chem. 267:6999-7006.

22. Nagley, P. 1991. Coordination of gene expression in the formation of mammalian mitochondria. TIG (Trends Genet.). 7:1-4

23. Nelson, B. D., A. Mutvei, and V. Joste. 1984. Regulation of biosynthesis of the rat liver inner mitochondrial membrane by thyroid hormone. Arch. Biochem. Biophys. 228:41-48.

24. Nelson, B. D. 1990. Thyroid hormone regulation of mitochondrial function. Comments on the mechanism of signal transduction. Biochim. Biophys. Acta. 1018:275-277.

25. Mutvei, A., and B. D. Nelson. 1989. The response of individual polypeptides of the mammalian respiratory chain to thyroid hormone. Arch. Biochem. Biophys. 268:215-220.

26. Koga, Y., M. Davidson, E. A. Schon, and M. P. King. 1993. Fine mapping of mitochondrial RNAs derived from the mtDNA region containing a point mutation associated with MELAS. Nucleic Acids Res. 21:657-662.

27. Reardon, W., R. J. M. Ross, and M. G. Sweeney. 1992. Diabetes mellitus associated with a pathogenic point mutation in mitochondrial DNA. Lancet. 340:1376-1379.

28. Rotig, A. 1992. Maternally inherited duplication of the mitochondrial genome in a syndrome of proximal tubulopathy, diabetes mellitus, and cerebellar ataxia. Am. J. Hum. Genet. 50:364-370.

29. Ballinger, S. W. 1992. Maternally transmitted diabetes and deafness associated with a $10.4 \mathrm{~Kb}$ mitochondrial DNA deletion. Nat. Genet. 1:11-15.

30. Gerbitz, K. D. 1992. Does the mitochondrial DNA play a role in the pathogenesis of diabetes? Diabetologia. 35:1181-1186.

31. Minchenko, A. 1988. Mitochondrial gene expression in rat liver and heart muscle in diabetes: effect of insulin and ribonucleotides. FASEB (Fed. Am. Soc. Exp. Biol.) J. 2:985a. (Abstr.)

32. Minchenko, A. G. 1981. Expression of the liver mitochondrial genome in diabetes. Probl. Encokrinol. 27:62-66.

33. Savabi, F., and A. Kirsch. 1991. Alteration of the phosphocreatine energy shuttle components in diabetic rat heart. J. Mol. Cell. Cardiol. 23:1323-1333.

34. Gross, M. D., S. Harris, R. E. Beyer. 1972. The effect of streptozotocininduced diabetes on oxidative phosphorylation and related reactions in skeletal muscle mitochondria. Horm. Metab. Res. 4:1-7.

35. Bjorntorp, P. 1966. Respiration and phosphorylation of mitochondria isolated from the skeletal muscle of diabetic and normal subjects. Diabetologia 2:346-352.

36. Mackerer, C. R., R. J. Paquet, M. A. Mehlman, and R. B. Tobin. 1971 Oxidation and phosphorylation in liver mitochondria from alloxan and streptozotocin diabetic rats. Proc. Soc. Exp. Biol. Med. 137:992-995. 